\section{Blood glucose and liver function in dogs administered a xylitol drinking water additive at zero, one and five times dosage rates}

\author{
James M.G. Anthony, ${ }^{1}$ Lynn P. Weber, ${ }^{2}$ \\ Stan Alkemade ${ }^{3}$ \\ 1Department of Small Animal Clinical \\ Sciences, Western College of Veterinary \\ Medicine,University of Saskatchewan, \\ Saskatoon; 2Department of Veterinary \\ Biomedical Sciences, Western College \\ of Veterinary Medicine, University of \\ Saskatchewan, Saskatoon; ${ }^{3 W}$ Worldwide \\ BioMedEx Inc., Arva, Ontario, Canada
}

\begin{abstract}
A study was designed to determine the safety of a drinking water additive that reduces plaque and calculus in dogs, and contains xylitol as an active ingredient. The randomized, double-blind, placebo-controlled study was performed in 15 crossbred dogs that were randomly divided into three groups and had their drinking water treated for 14 days with either: i) a commercial health care product (BreathaLyser Plus) at the recommended dosage, ii) an experimental health care product (BreathaLyser Plus containing five times the amount of xylitol), or iii) a placebo of purified water with a colour additive. Results demonstrated that the continuous administration of a commercial, drinking water, oral health product containing xylitol, at one and five times the normal inclusion rate, does not cause hypoglycemia or alter liver function in dogs.
\end{abstract}

\section{Introduction}

Xylitol is a five-carbon sugar alcohol that is used in a number of consumer products as a sweetener. The human consumption of this product has increased over the past few years. In animals, xylitol has proven to increase bone calcification, inhibit the growth of certain bacteria and prevents oral bacteria from producing the acids that demineralize the tooth surface. 1,2 There have been reports that dogs may develop mild to severe insulin induced hypoglycemia after ingesting products containing xylitol. ${ }^{3-5}$ Xylitol has little or no effect on plasma insulin or glucose level in humans, yet in dogs, xylitol increases insulin release and can cause severe hypoglycemia with ataxia, collapse and seizures. ${ }^{3}$ Although xylitol is absorbed slowly in humans, it is rapidly and almost fully absorbed in dogs, with peak plasma concentration occurring within $30 \mathrm{~min} .{ }^{6}$ Additionally, there have been reports that dogs may develop acute hepatic failure following xylitol ingestion. ${ }^{7}$ Therefore, since there are concerns regarding the safety of administering oral dental care products containing xylitol (BreathaLyser Plus) a study examining the toxicity in dogs is needed. To prove the safety of this product a 14 day oral toxicity study was conducted in dogs at the usual and a 5 times the recommended dosage in the Animal Research Centre at the Western College of Veterinary Medicine, Saskatoon, Saskatchewan, Canada.

\section{Materials and Methods}

A randomized, double blind, placebo controlled study was performed where fifteen crossbred dogs of mixed ages (mean years: 3.1 , range 2-7 years), weight (mean kg: 24.8, range 16-40) and sex (females: 8, males: 7) were randomly divided into three groups and their drinking water was treated for 14 days with either: i) commercial care product (BreathaLyser Plus, ImRex Inc., London, ON, Canada), ii) BreathaLyser Plus containing five times the amount of xylitol recommended by the manufacturer, or iii) placebo [purified water coloured to match solutions i) and ii)].

At the start of the study, there were no differences between the three groups for, sex, age, or weight. Throughout the trial, all dogs were maintained in the same environment and fed the same commercial dog food (Iams Adult, Iams Company, Dayton, OH, USA). No food supplementations such as treats or chew toys were given during the study. All dogs in this study were examined for general health and acclimatized to the facilities for eight days before entering the study. On day 0 , samples for complete blood count, and clinical chemistry [Sodium (Na), potassium (K), Na:K ratio, chlorine $(\mathrm{Cl})$, hydrogen tricarbonate ( $\mathrm{HCO} 3)$, Anion Gap, calcium (Ca), phosphorous (P), magnesium (Mg), urea, creatinine, glucose, total bilirubin, cholesterol, alkaline phosphatise (AP), alanine aminotransferase (ALT), gamma-glutamyltransferase (GGT), glutamate dehydrogenase (GLDH), creatine kinase (CK), total protein albumin, globulin, A:G ration, sorbitol dehydrogenase (SDH)] were collected at approximately $7 \mathrm{~h}$ after feeding. All samples were performed at an independent diagnostic laboratory (Prairie Diagnostic Services, Saskatoon, Saskatchewan, Canada). Each study group was then placed on drinking water containing the appropriate test product and maintained on the treated water for $24 \mathrm{~h}$ per day for 14 days. The principal investigator and
Correspondence: James M.G. Anthony,

Department of Small Animal Clinical Sciences, Western College of Veterinary Medicine, University of Saskatchewan, Saskatoon, Canada. E-mail: james.anthony@usask.ca

Key words: xylitol, toxicity, BreathaLyser Plus.

Conflict of interest: the authors report no conflicts of interest.

Received for publication: 19 January 2011. Accepted for publication: 11 April 2011

This work is licensed under a Creative Commons Attribution 3.0 License (by-nc 3.0).

(C) Copyright J.M.G. Anthony et al., 2010

Licensee PAGEPress, Italy

Veterinary Science Development 2011; 1:e2 doi:10.4081/vsd.2011.e2

all study and laboratory personnel were blind as to which test article was being administered to each group. Prior to feeding on the following day, each animal had blood collected for glucose analysis. Animals were fed and subsequent blood samples for glucose were obtained at two-hour intervals for a total of 4 samples for each animal. The animals were observed throughout the study period for any adverse effects or behavioural changes. On the last day of the study (day 14), blood samples were once again collected for complete blood count and blood chemistry at approximately $7 \mathrm{~h}$ after feeding, and the dogs were then returned to normal drinking water. Data are expressed as mean \pm standard error of the mean (SEM). Differences among treatment were tested using repeated measures analysis of variance (ANOVA) followed by Tukey's posteriori test, as appropriate. Differences were considered to be significantly different if $\mathrm{P}<0.05$.

\section{Results}

All dogs freely accepted and drank the water with the study additives. Throughout the study there were no noteworthy abnormal findings based on clinical blood chemistry. No adverse events were observed or reported. All glucose samples from all treatment groups were within the normal range during the entire study (Figure 1). Blood glucose showed a diel variation in all treatment groups on the first day of treatment, with blood glucose in the lower end of the normal range in the morning sample taken before feeding the dogs (Figure 1). The blood glucose then rose as the dogs fed throughout the rest of the day, but without any difference detected among treatment groups 
(Figure 1). However, there was a minor, but statistically significant increase in blood glucose at day 14 in the dogs from the 5 times treated group compared to the control group (Figure 1). Analyses of liver function tests (AP, ALT, GGT) demonstrated no significant effect of xylitol treatment at any time during the study (Table 1).

\section{Discussion}

Xylitol is a five-carbon sugar alcohol or pentitol that is mainly used as an artificial sweetener in a variety of human consumer products, such as sugar free chewing gum and baked goods. In animals (dogs and cats), xylitol is being used in some oral care products. Xylitol has an antibacterial effect on oral bacteria, especially on plaque, and decreases the formation and accumulation of calculus on the tooth. ${ }^{8-}$ 11 Oral xylitol has a wide margin of safety in most animal species. ${ }^{5}$ There have been reports in the literature that dogs may develop mild to severe insulin induced hypoglycemia after ingesting products containing xylitol. ${ }^{3-7}$ It has been suggested that dogs fed $0.1 \mathrm{~g} / \mathrm{kg}$ xylitol could develop hypoglycemia. ${ }^{7}$ This theory is based on the observation that dogs given intravenous xylitol demonstrated a dose dependant release of insulin that resulted in a concurrent drop in blood glucose.6,12,13 After a toxic dose of xylitol is ingested by dogs, the initial sign is vomiting, followed by lethargy, ataxia, collapse, and seizures. ${ }^{7}$ Hypoglycemia with hypokalemia (insulin causes potassium to move into cells with the glucose) and hypophosphatemia (insulin can increase cellular permeability to phosphate ions) is the physiological outcome. 5 Additionally, acute liver failure has been reported in dogs consuming a single large dose of xylitol; resulting in elevated liver enzymes: alanine transaminase (high), bilirubin (mild to moderate elevation), and alkaline phosphatase (mild). ${ }^{7}$ Thus, it is hypothesized, that a xylitol oral dosage of greater than $0.1 \mathrm{~g} / \mathrm{kg}$ can result in clinical sign of hypoglycemia and a dosage greater than $0.5 \mathrm{~g} / \mathrm{kg}$ can be hepatotoxic. ${ }^{5} \mathrm{We}$ hypothesize that at the recommended dosage of BreathaLyser Plus and at 5 times its dosage rate will not produce hepatotoxicity or hypoglycemia. This study of blood glucose and liver function in dogs administered a xylitol product at zero, one and five times dosage rates significantly demonstrated that BreathaLyser Plus is safe product even at 5 times the dosage rate in dogs. BreathaLyser Plus contains xylitol as an active ingredient. It is supplied as a concentrate which, when diluted according to the directions, produces drinking water containing $0.05 \mathrm{mg} / \mathrm{mL}$ of xylitol. Healthy dogs consume 20-70 $\mathrm{mL} / \mathrm{kg} /$ day of water, depending on the environmental temperature, physical activity, and whether they are fed moist or dry foods. ${ }^{14} \mathrm{~A}$ maximum intake of xylitol of $3.5 \mathrm{mg} / \mathrm{kg}$ at the recommended dosage and $17.5 \mathrm{mg} / \mathrm{kg}$ at five times the dosage would be consumed daily by each dog. Since the total daily water intake is not consumed at one time, the actual safety margins would be much higher. The current study of basic blood chemistry, blood glucose and liver function in dogs administered a xylitol product at 1 times and 5 times dosage demonstrated that BreathaLyser Plus is not hepatotoxic and does not cause hypoglycemia in dogs. In fact, the significant elevation in blood glucose at day 14 in the 5 times dosage group suggests that BreathaLyser Plus may in fact cause hyperglycemia, not hypoglycemia. However, it is

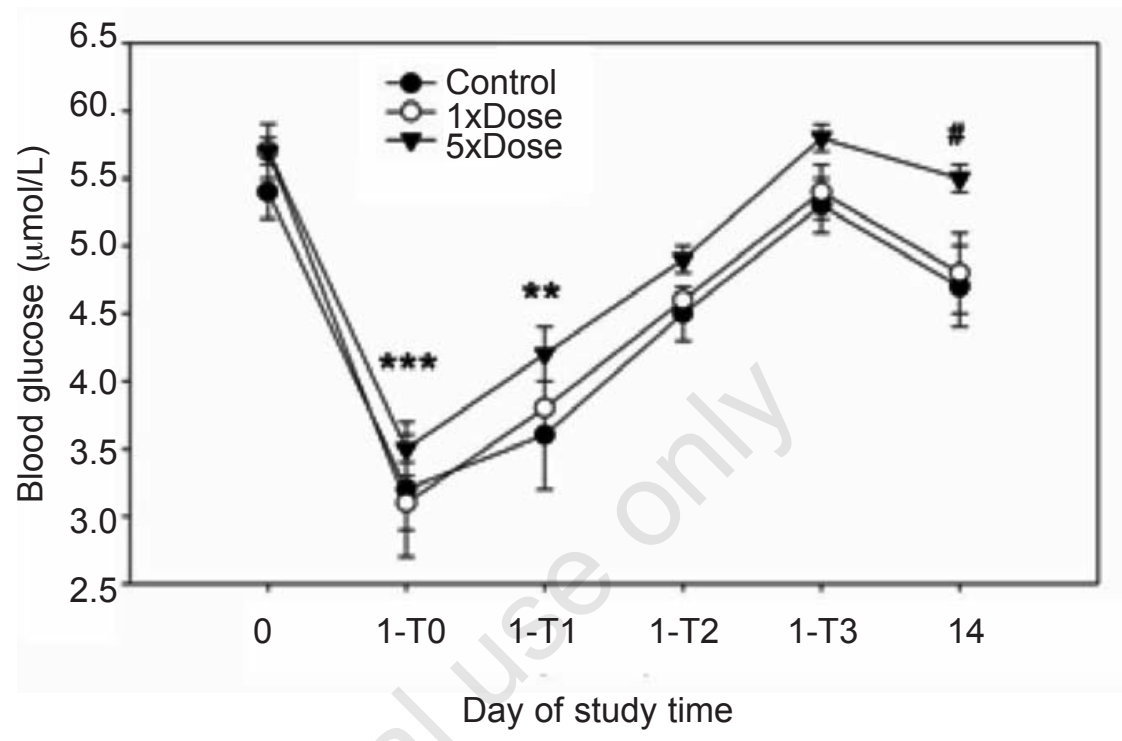

Figure 1. Blood glucose levels determined in dogs ( $n=5$ dogs per group) given normal tap water treated with a blue dye (control), water with the recommended dose of BreathaLyzer Plus (1x Dose) or water with Breathalyzer formulated to containing 5x xylitol ( $5 x$ Dose) for 14 days. Blood samples were taken from dogs one day before exposure (Day 0), at four time-points during the first day of exposure (Day 1 - T0, Day 1 - T1, Day 1 - T2 and Day 1-T3) and at the end of the exposure (Day 14). Results are shown as mean \pm SEM. Significant overall effects of treatment $(P=0.01)$ and time/day of blood collection $(\mathbf{P}<\mathbf{0 . 0 0 0 5})$ were detected using a two-way ANOVA. ${ }^{* *} \mathrm{P}<\mathbf{0 . 0 1},{ }^{* * *} \mathrm{P}<\mathbf{0 . 0 0 1}$ compared to Day 0 value within the same treatment group and $\# \mathbf{P}<0.05$ compared to control values at the same time point in Tukey's posteriori tests.

Table 1. Serum enzyme activities $(\mathrm{U} / \mathrm{L})$ in $\operatorname{dogs}(\mathrm{n}=5$ dogs per group) given normal tap water treated with a blue dye (control), water with the recommended dose of Breathalyzer Plus (1x Dose) or water with Breathalyzer formulated to containing $5 x$ xylitol (5x Dose) for 14 days. Blood samples were taken from dogs one day before exposure (Day 0 ) and at the end of the exposure (Day 14). Results are shown as mean \pm SEM. No significant effects of treatment were detected using a one-way repeated measure ANOVA.

\begin{tabular}{lccc} 
Serum Envayme & Group & Day 0 & Day 14 \\
ALT & Control & $56 \pm 9$ & $79 \pm 17$ \\
& 1x Dose & $37 \pm 2$ & $39 \pm 3$ \\
& 5x Dose & $136 \pm 56$ & $57 \pm 7$ \\
GLDH & Control & $4.8 \pm 0.6$ & $5.0 \pm 1.5$ \\
& 1x Dose & $2.4 \pm 0.4$ & $2.2 \pm 0.7$ \\
& 5x Dose & $10.0 \pm 6.0$ & $5.4 \pm 0.9$ \\
\hline CK & Control & $169 \pm 40$ & $388 \pm 96$ \\
& 1x Dose & $113 \pm 13$ & $288 \pm 70$ \\
& 5x Dose & $158 \pm 32$ & $140 \pm 20$ \\
SDH & Control & $0.0 \pm 0.0$ & $3.4 \pm 0.8$ \\
& 1x Dose & $0.0 \pm 0.0$ & $2.0 \pm 1.1$ \\
& 5x Dose & $0.0 \pm 0.0$ & $3.6 \pm 1.2$ \\
\hline
\end{tabular}

ALT, alanine aminotransferase; GLDH, glutamate dehydrogenase; CK, creatine kinase; SDH, sorbitol dehydrogenase. 
important to note that the blood glucose at this time, even though elevated, is still within the normal range. Therefore, based on the results of this study, BreathaLyser Plus is a safe product even at 5 times the dosage rate in dogs.

\section{References}

1. Cronin JR. Xylitol: a sweet for healthy teeth and more. Alternative Complementary Therapy 2003;9:139-41.

2. Gare F. The sweet miracle of xylitol. North Bergan, NJ, USA, Basic Health Publications Inc., 2003.

3. Dunayer EK. Hypoglycemia following canine ingestion of xylitol containing gum. Vet Hum Toxicol 2004;46:87-8.
4. Foss TS. Xylitol: sweet temptation for dogs. Vet Tech 2004;25:773-5.

5. Dunayer EK. New findings on the effects of xylitol ingestion in dogs. Vet Med-US 2006;Dec:791-6.

6. Kuzuya T, Kanazawa Y, Kosaka K. Stimulation of insulin secretion by xylitol in dogs. Endocrinology 1969;84:200-7.

7. Dunayer EK, Gwaltney-Brant SM. Acute hepatic failure and coagulopathy associated with xylitol ingestion in eight dogs. $\mathrm{J}$ Am Vet Med Assoc 2006; 229:1113-7.

8. Trahan L. Xylitol: a review of its actions on mutans streptococci and dental plaque-its clinical significance. Int Dent J 1995;45: 77-92.

9. Waler SM, Rolla G, Assev S, Clardi JE. The effect of xylitol on plaque metabolism. Swed Dent J 1984;8:155-61.
10. Makinen KK. New biochemical aspects of sweeteners. Int Dent J 1995;35:23-35.

11. Clarke DE. Drinking water additive decreases plaque and calculus accumulation in cats. J Vet Dent 2006;23:79-82.

12. Kuzuya T, Kanazawa Y, Kosaka K. Plasma insulin response to intravenously administered xylitol in dogs. Metabolism 1966; 15:1149-52.

13. Hirata Y, Fujisawa M, Sato H, et al. Blood glucose and plasma insulin responses to xylitol administered intravenously in dogs. Biophy Res Commun 1966;24:471-5.

14. Nelson RW. Polyuria, polydipsia and diabetes insipidus. Proceeding ACVIM 27th World Small Animal Veterinary Association Congress, Granada, Spain, 2002. 